mine the average grain size after crystallization. This could not be done with conventional JMAK analysis because it gives an overall crystallization rate wherein the nucleation and growth rates are coupled. Armed with the ability to determine the rates of nucleation and growth independently, the researchers found them to be constant. By plugging these values into a mathematical expression from the JMAK theory, the researchers found that the experimentally measured grain sizes and the mathematical predictions agree over a broad range of temperatures. This method can be used in practical applications to control grain size and materials properties that are strongly related to microstructure.

TAO XU

\section{Shock-Loading Strengthening Mechanism of Nanocrystalline Materials Revealed}

The Hall-Petch effect, in which the strength or hardness of crystalline materials increases inversely with the square root of their grain size, makes clear that grain boundaries (GBs) are natural obstacles for dislocation motions. The limitations of the dislocation motion cause the increase in the hardness and strength of the crystal materials. When the grain size approaches the nanoscale, this strengthening mechanism is no longer applicable, because the grain boundary itself is susceptible to motion (e.g., sliding). Also, the dislocation tends to be unstable at such a small grain size. High-strain-rate deformation experiments, as in shock-loading conditions, have been found to exhibit increased strength in nanocrystalline $\mathrm{Ni}$ and $\mathrm{Cu}$. However, the explanation of such a high strain rate $\left(10^{4} \mathrm{~s}^{-1}\right.$ to $\left.10^{6} \mathrm{~s}^{-1}\right)$ is still not clear, since the high strain rate is increased in conjunction with increasing pressure. Now, a team of researchers led by Eduardo M. Bringa at Lawrence Livermore National Laboratory (LLNL) and Helena Van Swygenhoven at the Paul Scherer Institute (Switzerland) has used molecular dynamics (MD) simulations as well as experimental methods to verify the underlying strengthening mechanism at such shock-loading conditions in nanocrystalline materials.

As reported in the September 16 issue of Science (p. 1838; DOI: 10.1126/science. 1116723), the researchers used MD simulations to systematically model shockloading behaviors during the plastic deformation processes of $\mathrm{Cu}$ nanocrystalline materials at different grain sizes (5-50 nm) and shockwave pressures (5-47 GPa). The MD simulations showed that with increasing hydrostatic pressure, a nanocrystalline metal $\mathrm{Cu}$ that deforms plastically by GB accommodation (e.g., GB sliding) becomes harder, due to the reduction of the GB sliding and the initialization/emergence of dislocations along the GB. The researchers also performed high-resolution electron microscope (HREM) experiments on Ni nanocrystals after shock-loading at $40 \mathrm{GPa}$. Ni was used for the experiments because $\mathrm{Ni}$ and $\mathrm{Cu}$ are $\mathrm{fcc}$ materials with similar shock impedances, but $\mathrm{Ni}$ has a larger stacking fault energy. The dislocation activity is clearly seen by HREM inside the grains, in excellent agreement with the MD simulation results.

FENGTING Xu

\section{Bioelectronic Nanodevices Fabricated on Live Bacteria}

Electrically percolating clusters of metal nanoparticles are ideal materials for electronic nanodevices because interparticle current occurs by single-electron transport. The negative surface charges of microorganisms such as viruses, yeasts, and bacteria, make them attractive scaffolds for templating metal nanoparticles. Now, researchers from the University of Nebraska-Lincoln have developed a method for building hybrid bioelectronic devices using gold nanoparticles and live bacteria. Furthermore, a biological response is used to control the electrical response of the devices.

As reported in an article published in the October 21 issue of Angerwandte Chemie, International Edition (p. 6668; DOI: 10.1002/ anie.200501711), V. Berry and R.F. Saraf deposited the gram-positive bacteria Bacillus cereus on a silica substrate containing linear gold electrodes $7 \mu \mathrm{m}$ apart, $10 \mathrm{~mm}$ long, and coated with poly(L-lysine) (PLL). Filtration and ultracentrifugation were used to extract from culture similar-sized bacteria-about 4-6 $\mu \mathrm{m}$ in length and $0.8-1.0 \mu \mathrm{m}$ in diameter. Typically, about 10 bridges formed along a pair of electrodes, which were $10 \mathrm{~mm}$ long, with a typical bridge composed of two bacteria. The researchers then immersed the bacteria-deposited chip in a solution containing PLL-coated gold nanoparticles (diameter = $30 \mathrm{~nm}$ ). Berry and Saraf said that the deposition of the gold nanoparticles, regulated simply by time, is highly selective with formation of a gold monolayer only on the negatively charged bacterial surface, because both the nanoparticles and the substrate are positively charged. The negatively charged teichoic acid (a polyelectrolyte on the bacterium surface) wraps the particle, screening the particle-particle repulsion and making the monolayer electrically percolating. A standard assay showed that the bacteria survived the fabrication process.
The researchers actuated the hydrophilic bacterial cell membranes by controlling the humidity, thereby modulating the separation between gold nanoparticles and concomitantly the interparticle electron tunneling current. Defining a device as a single bacterial bridge and measuring normalized current as a function of relative humidity, the researchers found that a decrease in humidity from $20 \%$ to $\sim 0 \%$ resulted in a $>40$-fold increase in tunneling current, even though the interparticle separation decreased by only $0.2 \mathrm{~nm}$. Berry and Saraf said, "We believe that such hybrids will be the key to conceptually new electronic devices that can be integrated with live microorganisms on flexible, plastic-like substrates by using simple chemistry."

STEVEN TROHALAKI

\section{Covalently Cross-linked Block Copolymer Micelles Encapsulate Magnetic Nanoparticles for Use in Biomedical Applications}

Magnetic nanoparticles are currently used in numerous biomedical applications in which stability and surface functionality are crucially important. In the October 5 issue of Nano Letters (p. 1987; DOI: 10.1021/nl0513939), B.-S. Kim, J.-M. Qiu, J.-P. Wang, and T.A. Taton from the University of Minnesota reported the encapsulation of magnetic nanoparticles $\left(\gamma-\mathrm{Fe}_{2} \mathrm{O}_{3}\right)$ within amphiphilic block copolymer micelles of poly(styrene 250 -block-acrylic $\left.\operatorname{acid}_{13}\right)$, which were subsequently crosslinked to secure the nanoparticles topologically. These "magnetomicelles" are stable to aggregation and have carboxylate groups on their surfaces that are readily modified.

The micelles, formed as a copolymer solution in $N, N$-dimethylformamide (DMF), were added to a second solution of nanoparticles in tetrahydrofuran (THF) in a defined ratio, forming a stable solution. Water was then slowly added to desolvate the copolymer core and nanoparticles simultaneously. Once cross-linked using a diamine cross-linker and a carbodiimide activator, the micelles offer a formidable defense against aggregation, changes in $\mathrm{pH}$, and chemical etching from $\mathrm{HCl}$ (a known etchant of $\gamma-\mathrm{Fe}_{2} \mathrm{O}_{3}$ nanoparticles). No degradation of the nanoparticle cargo occurred during cross-linking. In addition, Kim and co-workers were able to alter the surface chemistry of the micelles by adding $N$-ethyl- $N$ '-(3-dimethylaminopropyl)carbodiimide methiodide (EDC), N-hydroxylsulfosuccinimide sodium salt, and the fluorescent compound $\alpha$-amino- $\gamma-[5(6)$ carboxyamidofluorescein]-pentaethyleneglycol to the particle suspension. After this 